\title{
Pelvic dural ectasia leading to bilateral ureteric obstruction in the pediatric patient
}

\author{
Henry Tran, MD; ${ }^{*}$ Alex Kavanagh, MD, ${ }^{*}$ Paul Steinbok, MD, FRCSC; ${ }^{\dagger}$ Kourosh F. Afshar, MD, FRCSC
}

*Department of Urological Sciences, University of British Columbia, Vancouver, BC; 'Department of Pediatric Neurosurgery, University of British Columbia, Vancouver, BC

Cite as: Can Urol Assoc J 2013;7:E1 15-E1 17. hitp://dx.doi.org/10.5489/cuai.251

\section{Abstract}

Large pelvic cysts and moderate to severe hydroureteronephrosis were found after investigating hypertension in a 16-year-old child with Marfan's and known pelvic dural ectasia. Follow-up magnetic resonance imaging demonstrated extensive ectasia of the dural sac at the sacral level with displacement of the bowel, bladder and bilateral ureteric obstruction with accompanying hydroureteronephrosis that was advanced compared to prior imaging. Postural headaches secondary to cerebrospinal fluid leak and progressive hydroureteronephrosis prompted a combined neurosurgical and urologic resection of the pelvic masses. In this report, we discuss the preoperative evaluation and management of this rare form of bilateral ureteric obstruction.

\section{Case report}

Following aortic valve replacement and mitral valve repair, a 16-year-old female with Marfan's syndrome had a hypertensive crisis while under monitoring in the intensive care unit. Cardiac catheterization and aortograms were done which did not show any issues with the repair. A chest $x$-ray was performed the next day. An incidental finding of dilated and opacified renal collecting system was noted. This prompted an abdominal ultrasound revealing bilateral hydroureteronephrosis and a large cystic mass in the pelvis. Urology consult was requested for further management of the bilateral ureteric obstruction.

The patient remained entirely asymptomatic with normal bowel and bladder function. Her genitourinary exam was completely normal, except for mild urethral prolapse. Neurologic examination revealed no abnormality. The renal function remained normal (creatinine $57 \mu \mathrm{mol} / \mathrm{L}$ ). Magnetic resonance imaging (MRI) of her abdomen and thorax 1 year earlier for evaluation of her cardiac pathology revealed extensive ectasia of the dural sac at the sacral level (Fig. 1). This was determined to be an out-pouching through the S2 foramina on both sides into the presacral region as a large cystic area. No concominant hydroureteronephrosis was seen. A repeat MRI of the abdomen was performed showing enlargement of the pelvic cysts with bilateral hydroureteronephrosis (Fig. 2). Based on the progression of hydroureteronephrosis over the 1-year interval between scans and the development of hypertension, bilateral ureteric stents were inserted. This procedure was performed in an uncomplicated fashion and the patient was discharged from hospital.

Two months later, she returned to the hospital with complaints of progressive severe postural headaches. Neurosurgical evaluation prompted a meningomyelogram which revealed clear communication of the pelvic dural ectasia with the spinal subarachnoid space. There was also evidence of recent blood in the cyst. Her headaches were considered to be low pressure headaches, caused by cerebrospinal fluid leak into a partially ruptured pelvic meningocele. Bleeding into the cysts may have been facilitated by warfarin, an anticoagulant which she was taking because of her artificial heart valve. She consented to have her pelvic cysts resected and drained in a combined effort with urology and neurosurgery.

Cystoscopy revealed anteriorly displaced ureteric orifices and compression of the dome of the bladder. Ureteric catheters were placed and a midline laparotomy incision was made. The large pelvic cysts were immediately visible following division of the rectus sheath. The cecum and small bowel were displaced superiorly and the bladder was displaced inferiorly. Following dissection of the posterior peritoneum, the three ectatic dural sacs were clearly visible. Both ureters were identified and isolated from the cysts. 


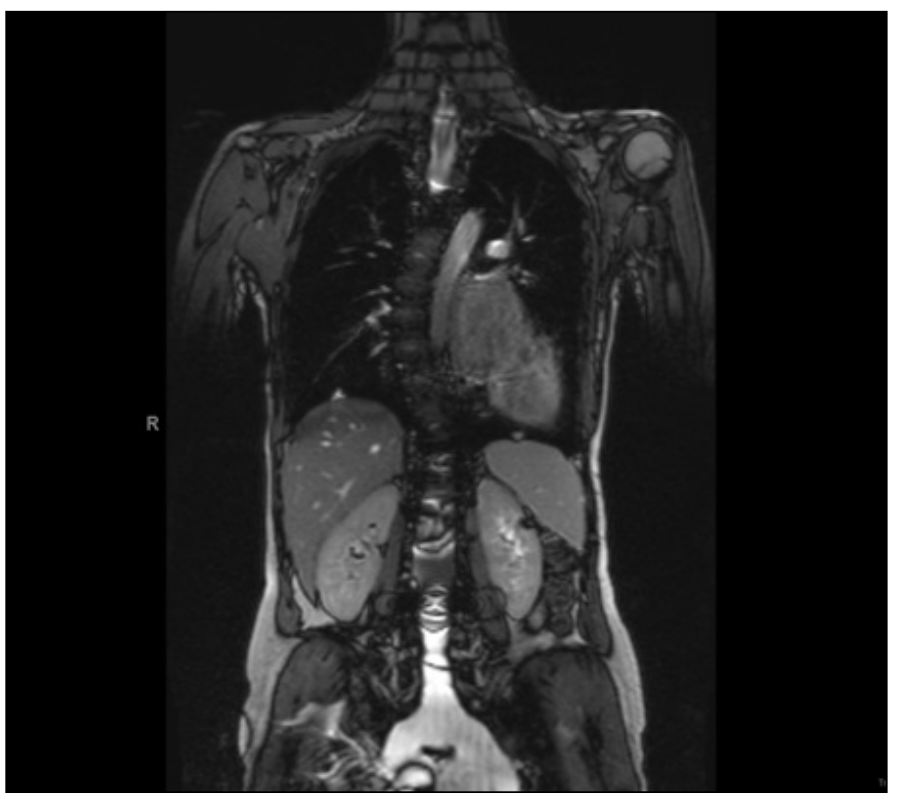

Fig. 1. A magnetic resonance image of the pelvis demonstrating two large ectatic dural sacs one year prior to presentation. No hydronephrosis.

The larger cyst was incised and clear fluid consistent with cerebrospinal fluid was drained. Sacral nerve roots were identified and determined to be free from the sac. The cyst was resected close to its origin from the nerve root foramen and closed in two layers with omental interposition. This procedure was repeated on the remaining two cysts and the abdomen was closed.

In the perioperative period, the patient developed a pseudomonas urinary tract infection, but her recovery was otherwise uncomplicated. Her postural headaches were eliminated following the procedure and she was discharged from hospital in stable condition. Follow-up renal ultrasound 1 month later showed resolution of the bilateral hydroureteronephrosis. Pathologic analysis of the resected cystic tissue revealed fibroconnective tissue with no evidence of meninges or central nervous system parenchyma.

\section{Discussion}

Dural ectasia is defined as an enlargement of the neural canal anywhere along the spinal column, usually in the lower lumbar and sacral regions. A number of radiographic features identifying dural ectasia have been suggested including a dilatation of the dural sac with the diameter of the thecal sac at S1 level greater than the diameter at L4 level,,$^{1,2}$ herniation of the dura along nerve root sleeves, and elevated dural sac ratios (the ratio between the dorsal sac diameter and vertebral body diameter at the same level). ${ }^{1}$ Despite these various radiographic features, no gold standard for diagnosis of dural ectasia has emerged. Dural ectasia is as a key characteristic of Marfan syndrome but may also be associated with Ehlers-Danlos syndrome, neurofibromatosis,

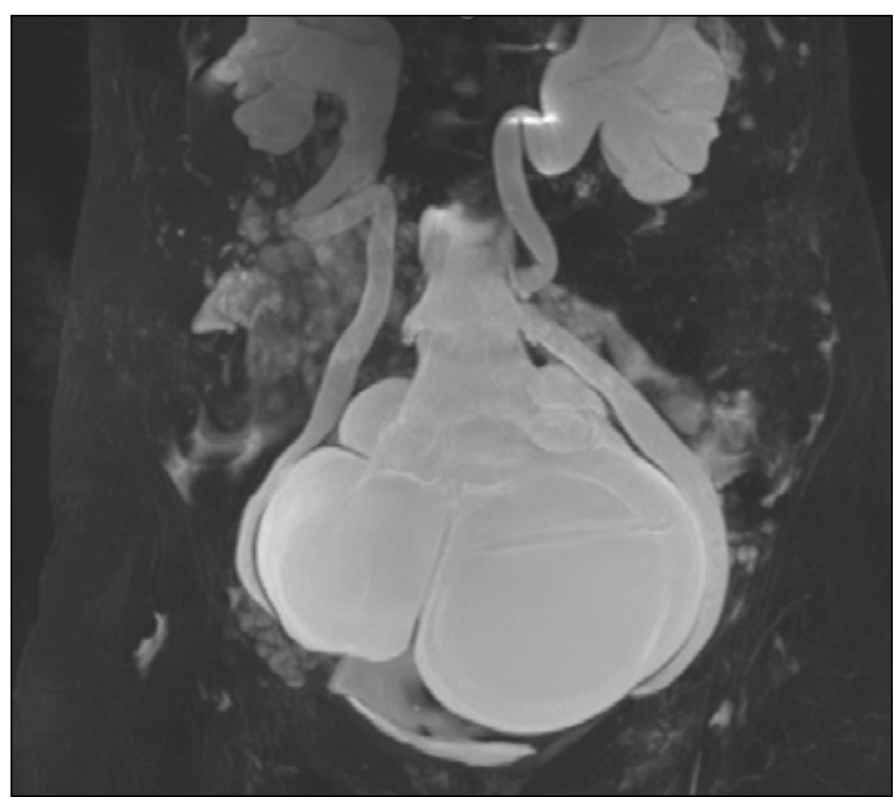

Fig. 2. A magnetic resonance urogram reconstruction of the pelvis demonstrating relationship of the large obstructing pelvic cysts to the ureters and kidneys.

ankylosing spondylitis and osteogenesis imperfecta tarda., ${ }^{1,2}$

Marfan syndrome is a connective tissue disorder of autosomal dominant inheritance, with an incidence of 1:5000. Ghent nosology is a set of clinical, radiologic and genetic criteria used in diagnosis of Marfan's syndrome. Revised 2010 Ghent nosology uses dural ectasia as one of the systemic features used in diagnostic scoring. It is hypothesized that in Marfan's syndrome, the dura mater is weaker and as a result, cerebrospinal fluid pulsations eventually lead to dural ectasia with gradual bone erosion. Most often, dural ectasia in Marfan's syndrome is subclinical and asymptomatic, however, large dural ectasias can be symptomatic. ${ }^{3}$ Three large studies have observed dural ectasia in $63-92 \%$ of patients with Marfan syndrome. ${ }^{1,4}$ The most common symptom is constipation, but other symptoms, including urinary retention or incontinence, dysmenorrhea, dyspareunia and pelvic or lower-back pain, can also occur from mass effects.,

The differential diagnosis for an obstructing pelvic cyst includes dural ectasias extending into the pelvis as an anterior sacral meningocele, as well as other masses of genitourinary, gynecologic and gastrointestinal origin including ovarian cysts, bladder diverticuli and congenital arachnoid cysts. ${ }^{4}$ Historically, large dural ectasias in the pelvis were treated using cyst aspiration via the rectum or vagina; however, this procedure was associated with a high morbidity and mortality from meningitis. ${ }^{5}$ Conservative management consisting of primarily observation is selected for patients with high surgical risk or in whom the dural ectasia is asymptomatic, small and unlikely to enlarge. Operative intervention is generally advocated in patients with symptomatic cysts causing pain, constipation, urinary retention or incontinence, or neurologic symptoms (such as postural 
headaches). Other indications for surgery include large size cyst due to risk of rupture, deteriorating renal function from ureteric or bladder outlet obstruction, and generalized mass effect on abdominal organs.

A single prior case report of dural ectasia causing urinary retention from bladder neck obstruction has been reported; however, to our knowledge, this is the first case report in the English literature where a dural ectasia in a patient with Marfan's syndrome caused hydroureteronephrosis from ureteric compression. The two methods of surgical intervention include a posterior approach by sacral laminectomy and an anterior transabdominal approach. The posterior approach involves a sacral laminectomy, followed by aspiration of the meningocele through the pedicle, and finally watertight closure of the pedicle. This approach is ideal for dural ectasias with a small meningocele pedicle and firm dura mater since watertight closure will be more easily attainable and there will be lower risk of cyst reformation. The anterior approach potentially allows for better closure of the pedicle since there is better exposure and the walls of both the pedicle and sac can be used for repair. ${ }^{7,8}$ The anterior approach is also the method of choice for emergency interventions; ${ }^{9}$ however, the anterior approach also carries more operative risk due to potential damage to the rectum with spillage of feculent material during separation of meningocele from the rectum, potential bleeding from epidural veins, and also greater infection risk from blood and serum in the presacral area. ${ }^{7,8}$ We considered using a posterior approach; however, because there were multiple large anterior cysts, we chose the anterior approach for easier accessibility and resection.

\section{Conclusion}

We present a rare form of bilateral ureteric obstruction due to a large anterior dural ectatic cyst. Due to a progression of the obstructing hydroureteronephrosis and neurologic sequelae of postural headaches, a resection of the pelvic cysts was done as a combined urological and neurosurgical procedure. Postoperatively, the child's headaches were eliminated. Early postoperative imaging demonstrates resolution of the prior hydronephrosis and her renal function remains stable.

Competing interests: None declared.

This paper has been peer-reviewed.

\section{References}

1. Lundby R, Rand-Hendriksen S, Hald J, et al. Dural ectasia in Marfan syndrome: a case control study. Am J Neuroradiology 2009;30:1534-40. http://dx.doi.org/10.3174/ajnr.A1620

2. Villeirs $G$, Van Tongerloo A, Verstraete $K$, et al. Widening of the spinal canal and dural ectasia in Marfan syndrome: assessment by CT. Neuroradiology 1999;41:850-4. http://dx.doi.org/10.1007/ s002340050856

3. De Paepe A. Dural ectasia and the diagnosis of Marfan's syndrome. Lancet 1999;354:878-9. hittp:// dx.doi.org/10.1016/S0140-6736(99)00168-3

4. Ho N, Hadley D, Jain P, et al. Case 47: dural ectasia associated with Marfan syndrome. Radiology 2002;223:767-71. http://dx.doi.org/10.1148/radiol.2233000971

5. Voyvodic F, Scroop R, Sanders R. Anterior sacral meningocele as a pelvic complication of Marfan syndrome. Aust NZ J Obstet Gynaecol 1999;39:262-5. http://dx.doi.org/10.1111/i.1479-828X.1999.tb03390.x

6. Sanchez A, Iglesias $C$, Lopez $C$, et al. Rectothecal fistula secondary to an anterior sacral meningocele. J Neurosurg Spine 2008;8:487-9. http://dx.doi.org/10.3171/SPI/2008/8/5/487

7. Tuzun Y, Izci Y, Yalcin Polat K. Anterior sacral meningocele: excision by abdominal approach. Ped Neurosurg 2005;41:244-7. http://dx.doi.org/10.1159/000087482

8. Ashley W, Wright N. Resection of a giant anterior sacral meningocele via an anterior approach: case report and review of the literature. Surg Neurology 2006;66:89-93. http://dx.doi.org/10.1016/i. surneu.2005.10.020

9. Bergeron E, Roux A, Demers J, et al. A 40 year-old woman with cauda equine syndrome caused by rectothecal fistula arising from an anterior sacral meningocele: case report. Neurosurgery 2010;67:E1 464-E1468. http://dx.doi.org/10.1227/NEU.0b013e3181f352ba

Correspondence: Dr. Kourosh Afshar, Department of Urological Sciences, University of British Columbia, Vancouver, BC; kafshar@cw.bc.ca 